\title{
LASCO/EIT Observations of Coronal Mass Ejections from Large-Scale Filament Channels
}

\author{
S.P. Plunkett ${ }^{1}$, K.P. Dere, R.A. Howard, D.J. Michels and G.E. Brueckner \\ E.O. Hulburt Center for Space Research, Naval Research Laboratory, \\ Washington, DC 20375, USA
}

\author{
B.J. Thompson ${ }^{2}$ \\ NASA Goddard Space Flight Center, Greenbelt, MD 20771, USA
}

\author{
J.-P. Delaboudinière \\ Institut d'Astrophysique Spatiale, Université Paris XI, 91405 Orsay \\ Cedex, France
}

\begin{abstract}
Solar activity in the latter half of 1996 was dominated by the presence of an extended filament channel in the southern hemisphere, forming a switchback configuration where it merged with the polar crown. Several large coronal mass ejections (CMEs) were observed to originate on or near this magnetic neutral line by the LASCO and EIT instruments on SOHO. As this channel gradually disappeared, a new, extended channel formed to the west of the old one, and this new channel also produced a number of large CMEs. We report here on observations of two events (December 23, 1996 and February 7, 1997), and discuss the global nature of these events.
\end{abstract}

\section{Introduction}

The dominant feature on the Sun in the latter half of 1996 (corresponding to the transition from cycle 22 to cycle 23) was an extended filament channel in the southern hemisphere, extending from close to the equator down to the polar crown. The channel was formed by the merger of the neutral line through an old, decaying active region at a latitude of about $S 25^{\circ}$ with the polar crown neutral line, forming a 'switchback' configuration. The resulting neutral line can clearly be seen on maps of the photospheric magnetic field recorded at the Wilcox Solar Observatory (http://quake.stanford.edu/“wso/synoptic.html). The emergence of new magnetic flux on Carrington Rotations (CR) 1916 and 1917 destroyed this magnetic configuration. During CR 1917, a new cycle active region emerged some $120^{\circ}$ west of the old switchback neutral line. The neutral line through this active region also merged with the polar crown neutral line, to form a new

\footnotetext{
${ }^{1}$ Universities Space Research Association

${ }^{2}$ Space Applications Corporation
} 
extended filament channel, again extending from close to the equator to the southern polar crown. This channel remained visible through the next several rotations.

These two filament channels produced a number of large coronal mass ejections (CMEs) that were observed in late 1996 and early 1997 by instruments on the Solar and Heliospheric Observatory ( $\mathrm{SOHO}$ ). In this paper, we present observations of two of these CMEs by the Large Angle Spectrometric Coronagraph (LASCO, Brueckner et al. 1995) and the Extreme Ultraviolet Imaging Telescope (EIT, Delaboudinière et al. 1995), showing for the first time the onset of and coronal response to a CME at EUV wavelengths.

\section{Observations}

\subsection{The December 23, 1996 Event}

This CME began with the activation and eruption of a short segment (about $35^{\prime \prime}$ long) of a prominence near an active region at heliographic location $\mathrm{S} 13^{\circ} \mathrm{W} 67^{\circ}$, observed at approximately 20:20 UT in the $195 \AA$ channel of EIT. This active region, and its associated filament, lay close to the position of the switchback filament channel described above. The event has been described in detail by Dere et al. (1997), so only a brief description will be given here.
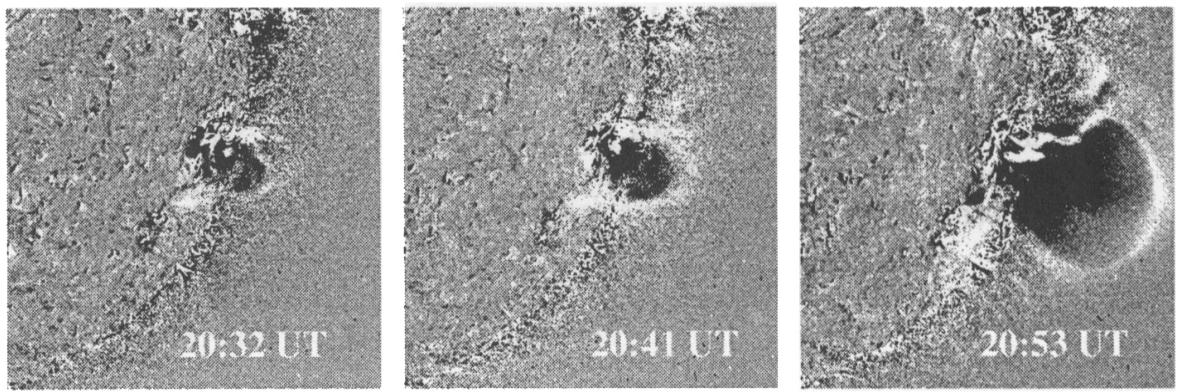

Figure 1. A sequence of running difference images in the Fe XII 195 $\AA$ passband of EIT, showing the onset of the December 23, 1996 CME.

Figure 1 shows a series of $195 \AA$ images recorded by EIT. The images are displayed as running differences, i.e., each image is shown as the difference from the preceding image in the series. This technique allows subtle changes in the coronal structure from one image to the next to be seen easily. A bright rim of emission, almost circular in appearance, surrounding a darker area, can be seen in the image at 20:32 UT, and is more clearly evident in the next two frames as it expands outwards. A complex, twisted structure that can be identified with the erupting prominence is visible within the dark 'cavity'. This three-part structure was also clearly observed by the LASCO C1 coronagraph at about 21:00 UT. The appearance of the CME in the $\mathrm{C} 2$ coronagraph is somewhat more complex. The C2 images show a bright loop-like structure moving out along a streamer, with a bright knot of prominence material following behind. The leading edge of the CME in $\mathrm{C} 2$ and in $\mathrm{C} 3 \mathrm{had}$ a projected speed of about $335 \mathrm{~km} \mathrm{~s}^{-1}$. 
A number of large scale phenomena accompanying the CME were observed by EIT. Several nearly simultaneous prominence activations were observed to the north and south of the CME launch site. Also, a large-scale wave motion propagated almost symmetrically outwards across the disk from the site of the CME, with a speed of about $100 \mathrm{~km} \mathrm{~s}^{-1}$. This wave, and other similar events observed by EIT, may be a coronal manifestation of Moreton waves observed in $\mathrm{H} \alpha$ in association with some large flares (Thompson 1998).

\subsection{The February 7, 1997 Event}
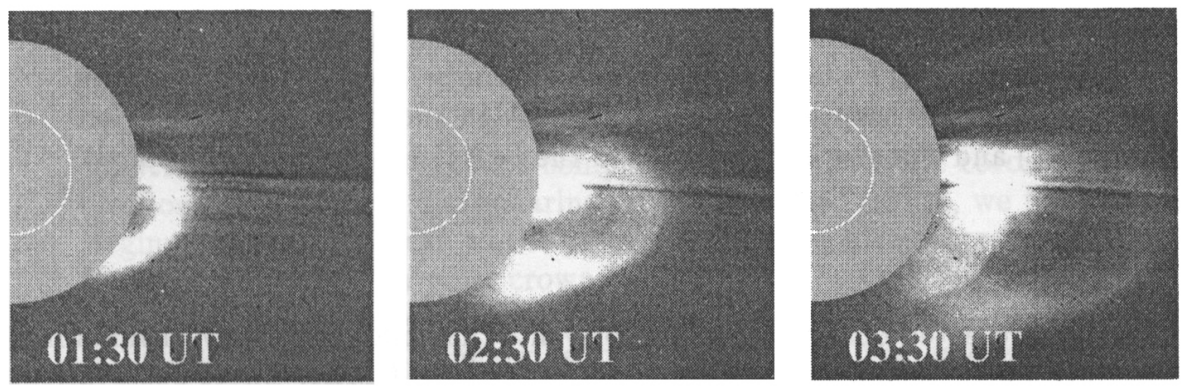

Figure 2. Development of the February 7, $1997 \mathrm{CME}$ as seen by LASCO C2. These are difference images, with a pre-event image subtracted from each image shown.

The partially Earth-directed CME of February 7, 1997 originated from the southwest quadrant of the Sun. The event has been discussed in some detail by Plunkett et al. (1997) and Gopalswamy (1997). LASCO observed the CME from about 01:00-10:00 UT in both $\mathrm{C} 2$ and $\mathrm{C} 3$ (see Figure 2). The event contained an arcade eruption, extending along the neutral line from close to the equator on the west limb, to the southern polar crown, and occupied the entire southwest quadrant in the LASCO images. Note that the neutral line involved in this event is not the same as that which produced the December 23 event described above; this event occurred some $120^{\circ}$ west of the earlier event. Bright prominence structures were clearly visible within the $\mathrm{CME}$, behind the frontal arcade. The leading edge of the CME was observed with an initial (projected) speed of about $170 \mathrm{~km} \mathrm{~s}^{-1}$ in $\mathrm{C} 2$ and accelerated to about $650 \mathrm{~km} \mathrm{~s}^{-1}$ by the time it reached $25 R_{\odot}$. A faint 'halo' component was also visible, extending to the south and east of the main arcade eruption.

The bright knot of prominence material seen in the LASCO images was clearly seen at the west limb in a He $304 \AA$ EIT image recorded at 02:07 UT (Figure 3). There was also a prominence eruption from the southwest limb over the same neutral line; this can be seen in Figure 3 as a series of faint, elongated structures off the southwest limb. A bright two-ribbon arcade structure was observed on the disk by EIT following the eruption of the CME. The footpoints of this arcade are clearly visible in the $195 \AA$ image shown in Figure 3, parallel to the neutral line from the equator to the polar crown. Images in the hotter $\mathrm{Fe}$ $\mathrm{XV} 284 \AA$ line show a set of sheared loops overlying these footpoints. 

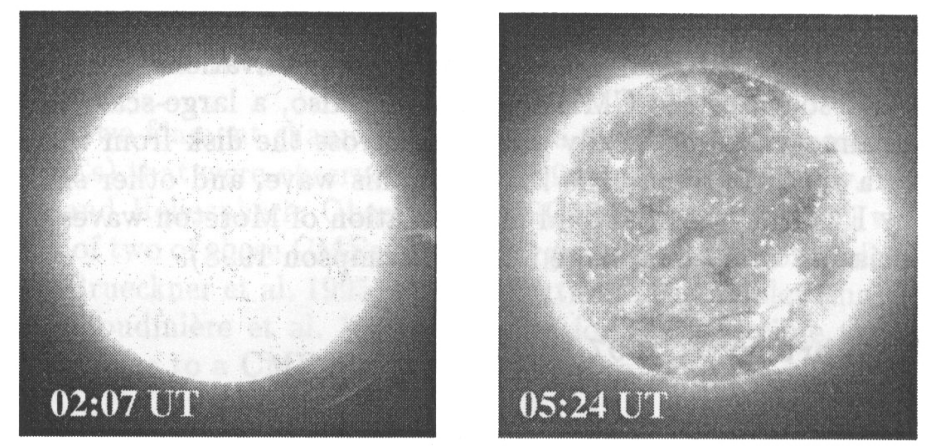

Figure 3. EIT observations of the February 7, $1997 \mathrm{CME}$, showing a prominence eruption to the W and SW in the He II $304 \AA$ line (left panel) and a post-ejection arcade in the Fe XII $195 \AA$ line (right panel).

\section{Conclusions}

We have presented observations of two events that offer some new insights into the CME phenomenon and confirm some ideas about CMEs. These observations allow us, for the first time, to study the onset of a CME on the solar disk at EUV wavelengths, and to study the coronal response to a CME in the same spectral region. The observations of the December $23 \mathrm{CME}$ show that the classical three-part structure (bright front, dark cavity and prominence core) of a CME is present right from the onset. The global nature of the CME is clear: in both events, a large fraction of the visible surface of the Sun is involved in the event. This is especially clear from the observations of prominence eruptions and arcade formation in the February 7 event. However, the December 23 observations show that a CME can begin in a very small volume, and that there is a propagation time delay before activity is observed in other regions of the corona.

Acknowledgments. We are grateful to our colleagues in the LASCO/EIT consortium for their support.

\section{References}

Brueckner, G.E. et al. 1995, Solar Phys., 162, 357

Delaboudinière, J.-P. et al. 1995, Solar Phys., 162, 291

Dere, K.P. et al. 1997, Solar Phys., 175, 601

Gopalswamy, N. 1997, SHINE Report on the February 7, 1997 CME

Plunkett, S.P. et al. 1997, in The Corona and Solar Wind Near Minimum Activity, ESA SP-404, ESA: Noordwijk, Holland, p. 615

Thompson, B.J. 1998, in preparation 